\title{
A Direct Measurement of the Neutron-Neutron Scattering Length
}

\author{
G. E. Mitchell ${ }^{1}$, W. I. Furman ${ }^{2}$, E. V. Lychagin ${ }^{2}$, A. Yu. Muzichka ${ }^{2}$, G. V. Nekhaev², \\ A. V. Strelkov², E. I. Sharapov², V. N. Shvetsov², B. G. Levakov³, V. I. Litvin ${ }^{3}$, A. E. \\ Lyzhin $^{3}$, E. P. Magda ${ }^{3}$, B. E. Crawford ${ }^{4}$, S. L. Stephenson ${ }^{4}$, C. R. Howell ${ }^{5}$, and W Tornow ${ }^{5}$ \\ ${ }^{1}$ North Carolina State University, Raleigh NC, \\ USA 27695-8202 and Triangle Universities Nuclear Laboratory, \\ Durham NC, USA 27708-0308 \\ ${ }^{2}$ Joint Institute for Nuclear Research, 141980 Dubna, Russia \\ ${ }^{3}$ Russian Federal Nuclear Center - All-Russian Research Institute of Technical Physics, \\ P.O. Box 245, 456770 Snezhinsk, Russia \\ ${ }^{4}$ Gettysburg College, Box 405, Gettysburg PA, USA 17325 \\ ${ }^{5}$ Duke University and Triangle Universities Nuclear Laboratory, \\ Durham NC, USA 27708-0308
}

\section{Received on 3 November, 2004}

\begin{abstract}
A direct measurement of $n n$-scattering by colliding free neutrons has never been performed. Indirect measurements continue to provide inconsistent results, leaving the issue of charge symmetry in the nuclear force unresolved. At present the Russian pulsed reactor YAGUAR is the best neutron source for such a measurement. A neutron moderator is installed in the central through channel and the scattered neutrons are detected at a distance of $12 \mathrm{~m}$ from the reactor. An instantaneous value of $1.1 \times 10^{18} / \mathrm{cm}^{2} \mathrm{~s}$ was obtained for the thermal neutron flux density. The experiment will be performed by the DIANNA Collaboration as ISTC project No. 2286.
\end{abstract}

\section{INTRODUCTION}

Values for the neutron-neutron ${ }^{1} \mathrm{~S}_{0}$ scattering length $a_{n n}$ appeared (by 1999) tohave converged to consistent numbers: $a_{n n}=-18.55 \pm 0.05$ (stat) \pm 0.3 (syst) fm [1] from the ${ }^{2} H\left(\pi^{-}, \gamma n\right) n$ reaction, and $a_{n n}=-18.7 \pm 0.3$ (stat) \pm 0.6 (syst) fm [2] from the ${ }^{2} H(n, n n) p$ reaction. Comparison with the well established value of the proton-proton scattering length $a_{p p}=-17.3 \pm 0.005$ (stat) \pm 0.4 (syst) fm indicates charge-symmetry breaking (CSB).

However, a recent investigation of the neutron-neutron and neutron-proton final-state interaction in the $n-d$ breakup reaction revived this issue. The $a_{n n}$-value of $-16.27 \pm 0.40 \mathrm{fm}$ was obtained in Ref. [3] for the ${ }^{2} H(n, n p) n$ reaction, where the total uncertainty consists of about equal parts of statistical and systematic errors. This latest result differs from that of Ref. [2] by almost $4 \sigma$, and renews the long-standing controversy regarding the conclusion on charge-symmetry breaking. An appealing solution is a direct measurement of the $n n$-scattering length. Such a measurement has been never performed. Here we describe the direct $n n$-scattering measurement which is under preparation at the unique aperiodic pulsed reactor YAGUAR.

\section{EXPERIMENTAL DESIGN}

For thermal neutrons the zero effective-range approximation works well. The effective thermal neutron cross section $\sigma$ measured with unpolarized neutrons is a statistical sum of the singlet $\sigma_{s}$ and triplet $\sigma_{t}$ cross sections. Since the Pauli exclusion principle for identical particles forbids the interaction of two neutrons in the triplet state, the cross section $\sigma_{t}$ is expected to be zero, and the measured cross section $\sigma$ is equal to $\pi a_{n n}^{2}$

Proposals for a direct measurement of the $n n$-scattering length have a long history; detailed references are provided in Ref. [4]. None of these proposals has been implemented. Except for the proposed use of underground nuclear explosions, most of the proposals are similar. The neutrons collide in a neutron interaction chamber, which we denote as the $n n$ cavity, and the scattered neutrons are detected externally. In such arrangements the "target" and the "beam" are neutrons produced by the same neutron source. Therefore, the $n n$ scattering intensity is proportional to the square of the neutron flux density, while the background intensity depends linearly on the flux density.

The situation is most favorable at pulsed neutron sources, where the thermal neutron flux density can be very high. It appears that the pulsed aperiodic reactor YAGUAR [5] at Snezhinsk, Russia, best fulfills requirements for the $n n$ cross section measurement. This $n n$ project was initially proposed at the Dubna ISINN-VIII meeting [6]. The fast neutron pulse is of $0.68 \mathrm{~ms}$ (FWHM) duration. The 40-liter volume of the YAGUAR active core contains the water soluted salt $\mathrm{UO}_{2} \mathrm{SO}_{4}$. The solution contains $465 \mathrm{~g}$ per liter of uranium which is enriched to $90 \%{ }^{235} \mathrm{U}$. The effective diameter of the cylindrical active core is $40 \mathrm{~cm}$, with an effective inside diameter of 17 $\mathrm{cm}$. The critical height is about $39 \mathrm{~cm}$, depending on the position of the startup rods. The body of the reactor has a central channel of $15-\mathrm{cm}$ diameter, which in the standard operating mode has startup rods that leave a cylindrical space of $12-\mathrm{cm}$ diameter.

A direct measurement of the $n n$-scattering length is to be performed using the time-of-flight (TOF) method by counting the scattered neutrons arriving after the reactor burst at a detector placed behind special collimators far away from the reactor. If only the colliding neutrons contribute to the 
detector counting rate, and if the parameters of the neutron field are determined and the geometry is known, then the detector counts (integrated over the thermal neutron part of the TOF spectrum), measure the $n n$-scattering cross section. The proposed arrangement for the $n n$-scattering experiment at YAGUAR is shown schematically in Fig. 1.

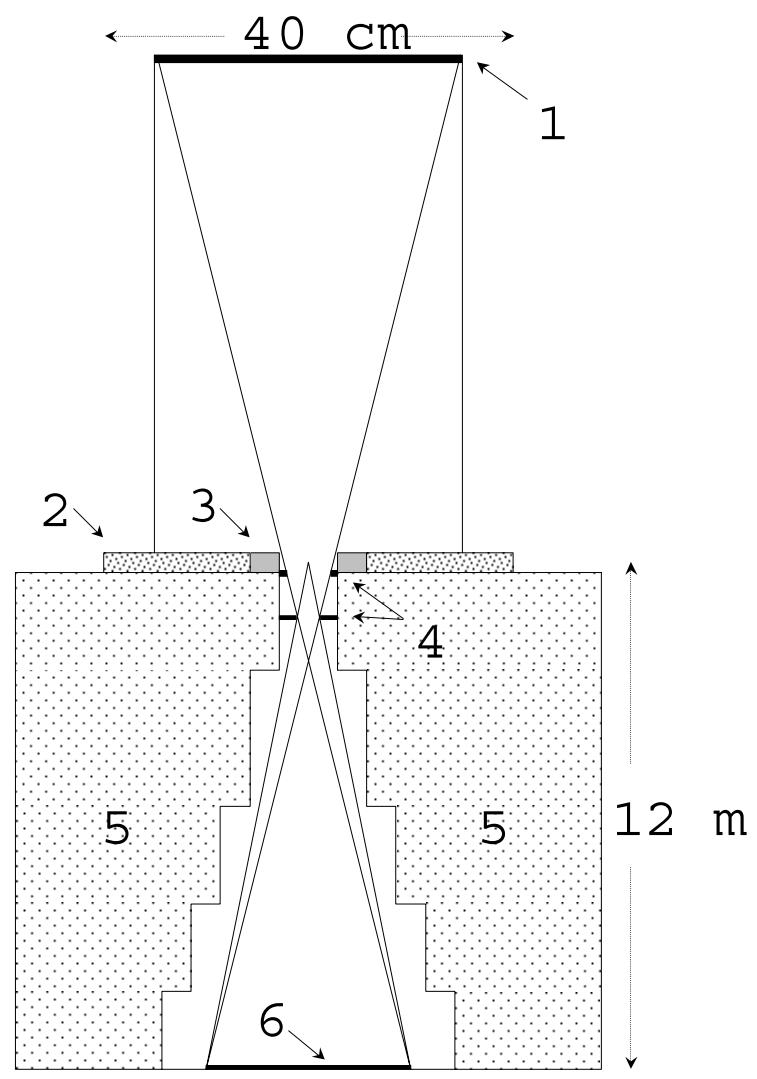

FIG. 1: Experimental setup (not to scale): (1) neutron absorber, (2) cylindrical reactor active core, (3) polyethylene moderator, (4) collimators, (5) shielding, (6) neutron detector.

The active core 2 is placed on three supports at $2 \mathrm{~m}$ above the floor level. The neutron $\mathrm{CH}_{2}$ moderator 3 is inserted inside the channel. An evacuated tube contains a collimation system 4 , and the neutron detector 5 is placed at a flight path of about $12 \mathrm{~m}$. The collimation system is designed to screen the source and to minimize background due to neutrons scattered from the walls. The ${ }^{3} \mathrm{He}$ absorber 1 reduces neutron scattering from the back wall.

\section{NEUTRON FLUX}

The reactor produces a hard neutron spectrum in the through-channel (average neutron energy $0.91 \mathrm{MeV}$ ) that is not suitable for the $n n$-experiment. We performed calculations for various moderators in the YAGUAR geometry and also performed measurements with various moderators at the reactor YAGUAR. The Monte Carlo modeling [7] was performed using the code MCNP-4 [8].

Neutron activation studies were performed to measure the thermal neutron densities inside cylindrical converters inserted into the YAGUAR reactor channel. The polyethylene converters are hollow cylinders with different inner diameters. The activation detectors for the absolute flux measurements were gold and copper foils placed in the central plane. Details of the measurements are given in [4]. The relative distribution of the neutron flux along the axis of the through-channel was also obtained. These measurements, combined with known neutron activation cross sections, determined the fluency, defined as the number of neutrons per $\mathrm{cm}^{2}$ per YAGUAR pulse.

The measured neutron fluencies for the four polyethylene converters vary linearly with thickness. This dependence agrees very well with Monte Carlo simulations [7]. With the experimental results for the fluence and using the value $\Delta t=0.7 \mathrm{~ms}$ for the thermal neutron pulse, we conclude that the 3-cm thick cylindrical moderator provides, for the pulse power of $30 \mathrm{MJ}$, an instantaneous value of $1.1 \times 10^{18} / \mathrm{cm}^{2} \mathrm{~s}$ for the thermal neutron flux $\Phi_{\text {central }}$ in the central region of the channel.

The moderated neutron flux spectrum is expected to be predominantly Maxwellian and to have a $1 / \mathrm{E}^{n}$ epithermal tail with the value of $n$ depending on the moderator thickness. The gold and copper activation data were analyzed to obtain experimental estimates of such parameters as the effective Maxwellian temperature, the relative size of the epithermal tail, and the slope parameter $n$.

\section{SIMULATIONS}

\section{A. Counting Rates}

After $n n$-scattering in the evacuated moderator channel, some of the scattered neutrons will reach a detector placed at about $12 \mathrm{~m}$ from the channel central plane, and will be measured by the TOF method with an effective solid angle $\Omega_{e f f}$. For ideal collimation, direct neutron paths from the moderator and the walls (besides the back wall of the $n n$ cavity) to the detector are excluded. Then the detector counts $N_{n n}$ per pulse (integrated over the thermal part of the TOF spectrum) are related to the $n n$-scattering cross section $\sigma_{n n}$, the average neutron flux density $\Phi_{a v}$, the effective pulse duration $\Delta t$, the effective $n n$-cavity volume $V$, and the most probable velocity $v_{0}$ by

$$
N_{n n}=2 c_{a v} \frac{\Phi_{a v}^{2}}{v_{o}} \sigma_{n n} \Delta t V \Omega_{e f f} \quad(\text { counts } / \text { pulse }),
$$

where the constant $c_{a v}$ is determined by Monte Carlo and analytical calculations for a given moderator geometry and neutron flux distribution. For a pure thermal neutron spectrum and including the CM/LAB transformation of the scattered flux in the direction of the detector, the value of $c_{a v}=$ $0.83 \pm 0.01$ [9]. Background considerations and the effects of fast-thermal and fast-fast collisions are discussed below. With 
a realistic value for the solid angle $\left(\Omega_{e f f} \simeq 5 \times 10^{-6}\right)$, then from Eq. (1) we expect $N_{n n} \simeq 180 n n$-counts in the neutron detector per burst.

\section{B. Backgrounds}

Ideally with extensive shielding no slow or fast source neutrons can reach the detector directly. However, in practice one can expect several background components.

The thermal neutron background component from the thermal and epithermal neutrons multiply scattered on collimators will depend on the collimation system. Several collimator options, as presented in [4] and in references therein, were considered, and an estimate of this component was found to be less than $20 \%$ even for the simplest two-diaphragm collimation.

Fast neutrons that originate during the reactor pulse are also separated by the TOF measurement. The time interval allocated for detecting thermal neutrons is about $5 \mathrm{~ms}$ and is delayed by $\simeq 2 \mathrm{~ms}$ relative to the maximum of the reactor pulse. At this time the subcritical reactor continues to emit fission neutrons. The background issues that these neutrons create were addressed in Ref. [10].

The background calculations and preliminary measurements of the background are very promising. More extensive modeling and detailed measurements are planned. Direct experimental separation of the $n n$-signal from the background is also possible, because the $n n$-scattering intensity is proportional to the square of the neutron flux density, while the background intensity depends linearly on the flux density.

\section{Time-of-Flight Spectra}

The $n n$ scattering measurements at the pulsed reactor YAGUAR will be performed by the TOF method. For a $12-\mathrm{m}$ flight path and $2.5-\mathrm{cm}$ thick cylindrical moderator the simulated 'incident' TOF spectrum is represented in Fig. 2 by the full curve. Such a spectrum would be measured by the neutron detector if it were placed as shown in Fig. 1 and the $n n$ cavity filled with a heavy gas such as argon, for which the transformation of the neutron spectrum after scattering can be neglected. The spectrum essentially consists of a Maxwellian part above $t=2 \mathrm{~ms}$ and a $1 / t^{2}$ epithermal part. For convenience we refer to the distributions above and below $t^{\star}=2 \mathrm{~ms}$ as the 'slow' and 'fast' components. This value of $t^{\star}$ is expected to be a cut off value for the experimental TOF spectrum below which the background will increase sharply.
The size of a possible contribution to the thermal energy region from 'fast-slow' and 'fast-fast' collisions producing 'slow' neutrons is addressed in Ref. [11]: the integral of the realistic scattered spectrum above $t^{\star}$ changes only slightly as compared to the pure Maxwellian case.

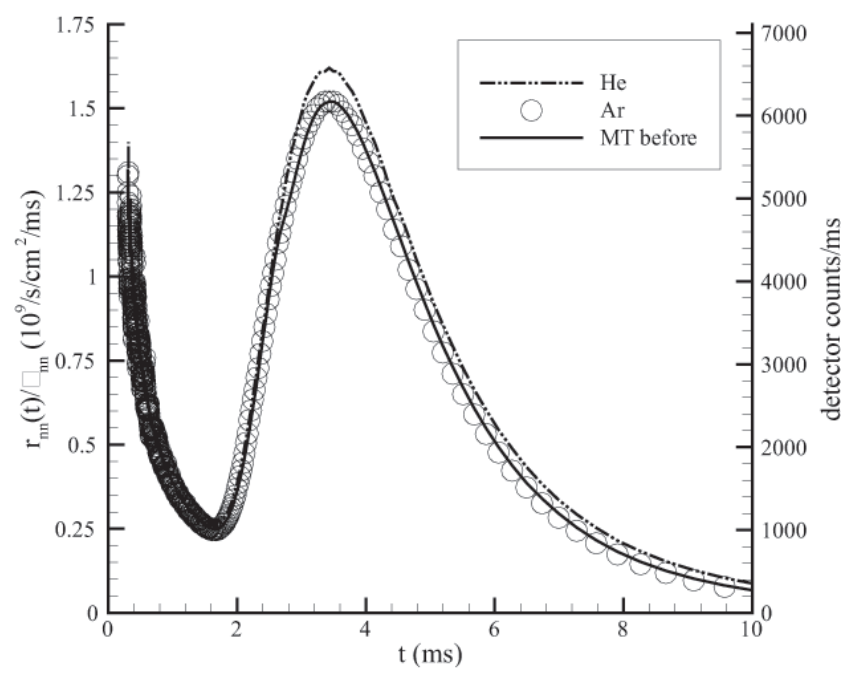

FIG. 2: The $\mathrm{n}$-gas collision rate $\left(\mathrm{r}_{n g}(\mathrm{t})\right.$ as a function of time of flight for $\mathrm{n}-\mathrm{He}$ scattering (dash-dot) and $\mathrm{n}$-Ar scattering (circle) compared with the initial flux scaled to the n-Ar spectrum (line). The right axis shows the estimated detector counts assuming a gas density of 0.2 Torr.

\section{SUMMARY}

An experiment to measure directly the $n n$ scattering length is at the late design stage. Measurements of the neutron flux and preliminary background measurements are consistent with simulations and indicate that the background contribution is of order $20 \%$. The final collimation arrangement will be installed shortly and initial measurements with the complete experimental system performed in 2005 .

\section{Acknowledgments}

This work was supported in part by the International Science and Technology Center under project No. 2286, by the Russia Foundation for Basic Research Grant No. 01-0217181, by the US DOE Office of High Energy and Nuclear Physics under grants DE-FG02-97-ER41042 and DE-FG0297-ER41033, and by the US NSF through an International Research Fellow Award No. 0107263.
[1] C.R. Howell et al., Phys. Lett. B 444, 252 (1998).

[2] D.E. González Trotter et al., Phys. Rev. Lett. 83, 3788 (1999).

[3] V. Huhn, L. Wätzold, Ch. Weber, A. Siepe, W. von Witsch, H. Witala, and W. Glöckle, Phys. Rev. C 63, 014003 (2001).
[4] W.I. Furman et al., J. Phys. G: Nucl. Part. Phys., 28, 2627 (2002).

[5] B.G. Levakov, N.V. Gorin, N.P. Kurakov, A.V. Lukin, A.E. Lyzhin, and N.N. Nevodnichy, in: Proceedings of the Topical 
Meeting on Physics, Safety and Application of Pulsed Reactors: Washington, D.C. (American Nuclear Society, La Grange Park, 1994), p. 67.

[6] C.D. Bowman, B.G. Levakov, A.E. Lyzhin, E.V. Lychagin, E.P. Magda, A.Yu. Muzichka, A.V. Strelkov, E.I. Sharapov, and V.N. Shvetsov, in: ISINN-VIII, Report E3-2000-192 (Joint Institute for Nuclear Research, Dubna, 2000), p. 245.

[7] S.L. Stephenson et al., in: ISINN-X, Report E3-2003-10 (Joint Institute for Nuclear Research, Dubna, 2003), p. 427.

[8] J.F. Briesmeister (Ed.), "MCNP ${ }^{T M}$ - A General Monte Carlo N-Particle Transport Code, Version 4c", Report LA-13709-M
(Los Alamos National Laboratory, Los Alamos, 2000).

[9] B.E. Crawford et al., in: ISINN-X, Report E3-2003-10 (Joint Institute for Nuclear Research, Dubna, 2003), p. 436.

[10] G.P. Gueorguiev, C.R. Howell, G.E. Mitchell, E.I. Sharapov, W. Tornow, in: Proceedings of the Sixth International Topical Meeting on Nuclear Applications of Accelerator Technology, AccApp'03 (American Nuclear Society, Inc., La Grange Park, 2004), p. 53.

[11] B.E. Crawford et al., J. Phys. G: Nucl. Part. Phys. 30, 1269 (2004). 